\title{
$-\mathrm{NOTES}-$
}

\section{ON THE ASYMPTOTIC STABILITY OF OSCILLATORS WITH UNBOUNDED DAMPING*}

\author{
BY ZVI ARTSTEIN AND E. F. INFANTE (Brown University)
}

\begin{abstract}
Through a technique inspired by the invariance principle of LaSalle, a general growth condition on the damping coefficient $h(t)$ of the equation

$$
\ddot{x}+h(t) \dot{x}+k x=0, k>0, h(t) \geq \epsilon>0,
$$

is given which is sufficient for the global asymptotic stability of the origin yet permits this coefficient to grow to infinity with time. The methods used do not depend on linearity, and are applied to obtain similar results to the nonlinear analogue of this equation.
\end{abstract}

1. Introduction. The differential equation

$$
\ddot{x}+h(t) \dot{x}+k x=0,
$$

with $h(t) \geq 0, k>0$, and its nonlinear generalizations are basic mathematical models for the representation of damped oscillatory phenomena; the global asymptotic stability of the rest point $x=\dot{x}=0$ of this equation and its generalizations has drawn the attention of numerous investigators (see, for example, $[1,2,3]$ ). It is well known that if the damping coefficient $h(t)$ is nonnegative, then this rest point is stable. If the damping coefficient is restricted to $0<\underline{h} \leq h(t) \leq \bar{h}, \underline{h}, \bar{h}$ constants, then the rest point is globally asymptotically stable; on the other hand, it is known through examples $[1,5]$ that if the damping coefficient is not bounded above, then the rest point, although stable, is not necessarily asymptotically stable and solutions may exist such that $x(t) \rightarrow c \neq 0$ and $\dot{x}(t) \rightarrow 0$ as time increases.

It is possible to give a physical interpretation for this behavior. The total energy of the system $V=\frac{1}{2} \dot{x}^{2}+\frac{1}{2} k x^{2}$ is dissipated at the rate $\dot{V}=-h(t) \dot{x}^{2}$; if $h(t) \geq \underline{h}>0$, all the kinetic energy $\frac{1}{2} \dot{x}^{2}$ will surely be dissipated from the system; this is not necessarily so for the potential energy. If the damping coefficient is also bounded above, then it is relatively simple to show that all of the total energy will be dissipated; the rest point $x=\dot{x}=0$ is then globally asymptotically stable. If $h(t)$ is unbounded, this is no longer necessarily the case; if the damping coefficient grows sufficiently fast, then the asymptotic state obtained can be one in which the system retains positive potential energy indefinitely and, therefore, the rest point is no longer globally asymptotically stable.

This phenomenon is of physical interest; there is a number of realistic situations that can be represented by Eq. (1.1), or its generalizations, where the damping coefficient increases without bound. Examples from tempering and heat treatment of materials

* Received August 4, 1975. This research was supported in part by the National Science Foundation under GP 28931X3, and in part by the Office of Naval Research under NONR N1467-AD-101000907. 
and of poisoning of catalysts are in this category. It is of interest to investigate, in such cases, the asymptotic state of the system.

The purpose of this note is to present a simple growth condition for the positive damping coefficient $h(t)$ which is sufficient to ensure the global asymptotic stability of the rest point, yet permits this coefficient to grow to infinity with time. It is somewhat surprising that, given the wealth of results known about equations of this type, no such condition or type of condition seems to be available in the literature.

The technique used here is inspired by the invariance principle of LaSalle $[4,5]$, as we explain in what follows.

The sufficient condition given can be made more precise and can as well be generalized to a wider class of equations. We wish to restrict ourselves to showing, through a counterexample, that if the stated sufficient condition is not met, the rest point is not globally asymptotically stable, and to indicating that similar results hold for the nonlinear equation

$$
\ddot{x}+h(x, \dot{x}, t) \dot{x}+f(x)=0,
$$

with $h \geq 0$ and $x f(x) \geq 0$.

2. The main result. Consider Eq. (1.1) written in its equivalent form

$$
\begin{aligned}
& \dot{x}=y \\
& \dot{y}=-k x-h(t) y .
\end{aligned}
$$

To obtain a growth condition result, the following lemma is useful

Lemma. Let $a_{1}, a_{2}, \cdots$ be a sequence of positive numbers with the property that, for some $N_{0}, B$ fixed, $\sum_{i=1}^{k} a_{i} \leq\left(k+N_{0}\right)^{2} B$ for all $k$. Then

$$
\sum_{i=1}^{\infty} \frac{1}{a_{i}}=\infty \text {. }
$$

Proof: It is easy to show (induction, or Cauchy-Schwartz) that if $b_{1}, \cdots, b_{m}$ are positive and $\sum_{i=1}{ }^{m} b_{i} \leq L$, then

$$
\sum_{i=1}^{n} \frac{1}{b_{i}} \geq m^{2} / L
$$

Let now $b_{i}=a_{2^{n+i}}, i=1, \cdots, 2^{n}$. From the assumption stated, it follows that $\sum_{i=1}{ }^{2^{n}} b_{i}$ $\leq\left(2^{n+1}+N_{0}\right)^{2} B$ and, therefore

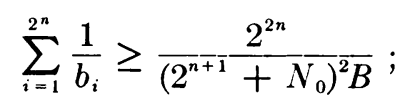

but this lower bound tends to $1 / 4 B$ as $n \rightarrow \infty$ and

$$
\sum_{i=1}^{\infty} \frac{1}{a_{i}}=\frac{1}{a_{i}}+\sum_{n=0}^{\infty} \sum_{i=1}^{2^{n}} \frac{1}{a_{2^{n}+i}} .
$$

The main result now follows:

Theorem. Suppose that $h(t)$ in (2.1) satisfies $h(t) \geq \underline{h}>0$ for all $t$ and that, for all $T \geq 0$ and some $B$, the growth condition

$$
\frac{1}{T^{2}} \int_{0}^{T} h(t) d t \leq B
$$

holds. Then every solution $(x(t), y(t))$ of $(2.1)$ approaches $(0,0)$ as $t \rightarrow \infty$. 
Proof: Let $(x(t), y(t))$ be a solution. Consider the natural Liapunov function $V(x, y)$ $=\left(y^{2} / 2\right)+k\left(x^{2} / 2\right)$; then $V(t)=V(x(t), y(t))$ satisfies $d V / d t=-h(t) y^{2}(t)$, and it follows that $V(t)$ is nonincreasing and nonnegative. Hence, $V(t) \rightarrow k c^{2} / 2$, for some $c$, as $t \rightarrow \infty$; in particular, the solution $(x(t), y(t))$ is positively bounded and approaches the ellipse $\left(y^{2} / 2\right)+k\left(x^{2} / 2\right)=k\left(c^{2} / 2\right)$. On the other hand, $d V / d t \leq-\underline{h} y^{2}$, so that any limit point of $(x(t), y(t))$ must have the form $(c, 0)$ (compare with [6]).

We claim that there is a monotonic increasing sequence of integers $n_{1}, n_{2}, \cdots$, $n_{i} \rightarrow \infty$ as $i \rightarrow \infty$, such that

$$
\int_{n_{i}}^{n_{i}+1} h(t) y(t) d t \rightarrow 0, \quad i \rightarrow \infty .
$$

Suppose the contrary; then there exist a $\delta>0$ and $n_{0}$ such that

$$
0<\delta \leq\left(\int_{n}^{n+1} h(t) y(t) d t\right)^{2}, \text { for } n \geq n_{0} ;
$$

it then follows (Cauchy-Schwartz inequality) that

$$
\delta \leq \int_{n}^{n+1} h(t) d t \int_{n}^{n+1} h(t) y^{2}(t) d t,
$$

and, setting $a_{n}=\int_{n}^{n+1} h(t) d t$, that

$$
\delta \frac{1}{a_{n}} \leq V(n)-V(n+1)
$$

summation for $n \geq n_{0}$ yields

$$
\delta \sum_{n=n_{\mathrm{o}}}^{\infty} \frac{1}{a_{n}} \leq V\left(n_{0}\right)
$$

which implies that

$$
\sum_{i=n_{0}}^{\infty} \frac{1}{a_{i}}<\infty .
$$

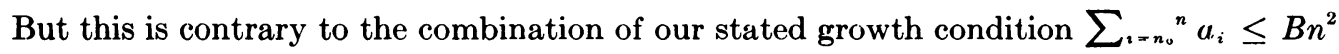
and our Lemma. Hence, (2.2) is true.

Integrate now the second of Eqs. (2.1) over $\left[n_{i}, n_{i}+1\right]$ to obtain

$$
y\left(n_{i}+1\right)-y\left(n_{i}\right)=-k \int_{n_{i}}^{n_{i}+1} x(t) d t-\int_{n_{i}}^{n_{i+1}} h(t) y(t) d t ;
$$

as $i \rightarrow \infty$, application of (2.2) yields $0=-c$ which rules out the possibility $c \neq 0$, thus completing the proof.

It is clear from the proof that the growth condition on $h(t)$ need be satisfied only on one sequence of subintervals rather than on the whole line. For instance, the growth condition

$$
\frac{1}{m^{2}} \sum_{i=1}^{m} \int_{n_{i}}^{n_{i}+1} h(t) d t \leq B,
$$

for some $B$ and all $m$, for one sequence of integers $\left\{n_{i}\right\}, n_{i} \rightarrow \infty$ as $i \rightarrow \infty$, is sufficient for asymptotic stability. 
3. The relation to the invariance principle. The proof of our theorem is fundamentally based on the LaSalle invariance principle [4-6], whose basic idea we wish to note here, since its application should be fruitful in the study of equations more general than the one investigated here.

It will be noted that, on the interval $\left[n_{i}, n_{i+1}\right]$, the solution $(x(t), y(t))$ of $(2.1)$ satisfies the differential equation

$$
\begin{aligned}
& \dot{x}=y \\
& \dot{y}=-k x-g(t)
\end{aligned}
$$

where $g(t)=h(t) y(t)$. As $i \rightarrow \infty$, the initial conditions $\left(x\left(n_{i}\right), y\left(n_{i}\right)\right)$ converge to $(c, 0)$ for some $c$ and (3.1) tends, asymptotically, by (2.2), to

$$
\begin{aligned}
& \dot{x}=y \\
& \dot{y}=-k x .
\end{aligned}
$$

The convergence of (3.1) to (3.2) implies continuous dependence with respect to initial data; in particular, $(c, 0)$ should be an equilibrium point for (3.2), but it obviously is not unless $c=0$.

This device is the basis for the invariance principle [7-9] for nonautonomous equations. It must be remarked, however, that the original equation does not have (3.2) as a limiting equation: it is along the solution that (2.1) is equivalent to (3.1) which converges to (3.2). A similar idea was used in [5].

4. Further remarks. We wish to indicate briefly how to extend our result to the nonlinear equation (1.2).

Typically, we assume that

(i) $x f(x)>0$ for $x \neq 0$, that

(ii) $\int_{0}^{x} f(z) d z \rightarrow \infty$ as $|x| \rightarrow \infty$, and

(iii) $h(x, \dot{x}, t) \geq w(x, \dot{x})$,

where $w$ is continuous and nonnegative and $w(x, y)>0$ for $y \neq 0$; it follows that $V(x, y)$ $=y^{2} / 2+\int_{0}^{x} f(z) d z$ is an appropriate Liapunov function from which, as in the Theorem, we conclude that any solution $(x(t), y(t)) \rightarrow(c, 0)$. The conclusion of the theorem follows upon the imposition of the growth condition

$$
\frac{1}{T^{2}} \int_{0}^{T} h(x(t), y(t), t) d t \leq B
$$

for some $B$ and all $T \geq 0$ and $(x(t), y(t))$ in a compact set.

Finally, we wish to remark on the growth condition stated in the Theorem.

The assumption of boundedness for all $T$ of

$$
\frac{1}{T^{2}} \int_{0}^{r} h(t) d t
$$

implies that, in a sense, $h(t)$ grows no more than linearly in time. In particular, if $h(t) \leq K+M t$, this growth condition is satisfied. This result is sharp in the sense that the $1 / T^{2}$ cannot be replaced by $1 / T^{2+\epsilon}$ for any $\epsilon>0$; alternatively, that $h(t) \leq$ 
$K+M t^{1+\epsilon}$ does not assure global asymptotic stability. Indeed, let $\epsilon>0$ and

$$
h(t)=(\epsilon+1) t^{-1}+\frac{1}{\epsilon} t+\frac{1}{\epsilon} t^{1+\epsilon} .
$$

Then $(x(t), y(t))=\left(1+t^{-\epsilon},-\epsilon t^{-\epsilon-1}\right)$ satisfies $\dot{x}=y, \dot{y}=-x-h(t) y$, but $(x(t), y(t)) \rightarrow$ $(1,0)$ as $t \rightarrow \infty$.

Similar examples have been produced by Levin and Nohel [1] and LaSalle [5].

\section{REFERENCES}

[1] J. J. Levin and J. A. Nohel, Global asymptotic stability of nonlinear systems of differential equations and applications to reactor dynamics, Arch. Rat. Mech. Anal. 5, 194-211 (1960)

[2] T. A. Burton and J. W. Hooker, On solutions of differential equations tending to zero, J. Reine Angew. Math. 267, 151-165 (1974)

[3] N. Onuchic, Invariance properties in the theory of ordinary differential equations with applications to stability problems, SIAM J. Control 9, 97-104 (1971)

[4] J. P. LaSalle, The extent of asymptotic stability, Proc. Nat. Acad. Sci. 46, 363-365 (1960)

[5] J. P. LaSalle, An invariance principle in the theory of stability, in Differential equations and dynamical systems, eds. J. K. Hale, and J. P. LaSalle, Academic Press, 1967, pp. 277-286

[6] J. P. LaSalle, Stability theory for ordinary differential equations, J. Diff. Equations 9, 57-65 (1968)

[7] I. R. Wakeman, An application to topological dynamics to obtain a new invariance property for nonautonomous ordinary differential equations, J. Diff. Equations 17, 259-295 (1975)

[8] J. P. LaSalle, Stability theory and invariance principles, in Proc. international symposium on dynamical systems, Brown University, August 1974, Academic Press (to appear)

[9] Z. Artstein, The limiting equations of nonautonomous ordinary differential equations, J. Diff. Eqs. (to appear) 